Supporting Information

\title{
Reflected laser interferometry: A versatile tool to probe condensation of low surface tension droplets
}

Sirshendu Misra ${ }^{\S}$, Hideaki Teshima†, Koji Takahashi†, ${ }^{\dagger}$, Sushanta K. Mitra* ${ }^{*} \S$

$\S$ Micro \& Nano-Scale Transport Laboratory, Waterloo Institute for Nanotechnology, Department of Mechanical and Mechatronics Engineering, University of Waterloo, Waterloo, 200 University Avenue West, Waterloo, Ontario N2L 3G1, Canada

$\dagger$ Department of Aeronautics and Astronautics, Kyushu University, Nishi-Ku, Motooka 744 Fukuoka 819-0395, Japan

$\$$ International Institute for Carbon-Neutral Energy Research (WPI-I2CNER), Kyushu University, Nishi-Ku, Motooka 744 Fukuoka 819-0395, Japan

*To whom correspondence should be addressed: E-mail: skmitra@uwaterloo.ca. 


\section{List of supporting videos}

Supporting video S1: Dual-wavelength interferograms capturing condensation of acetone microdroplets on quartz surface - observation set \#1 (MP4)

Supporting video S2: Dual-wavelength interferograms capturing condensation of acetone microdroplets on quartz surface - observation set \#2 (MP4) 


\section{Supplementary note 1}

\section{Time evolution of a condensing acetone microdroplet on quartz surface - observation set \#2}

In addition to the observation set \#1 (Figure 3) reported in the main manuscript, we analyze another set of data from the same experiment where we target a different droplet and track its evolution as it grows and undergoes multiple coalescence due to condensation. The evolution of the droplet is represented in the form a discrete timeseries in Figure S1.

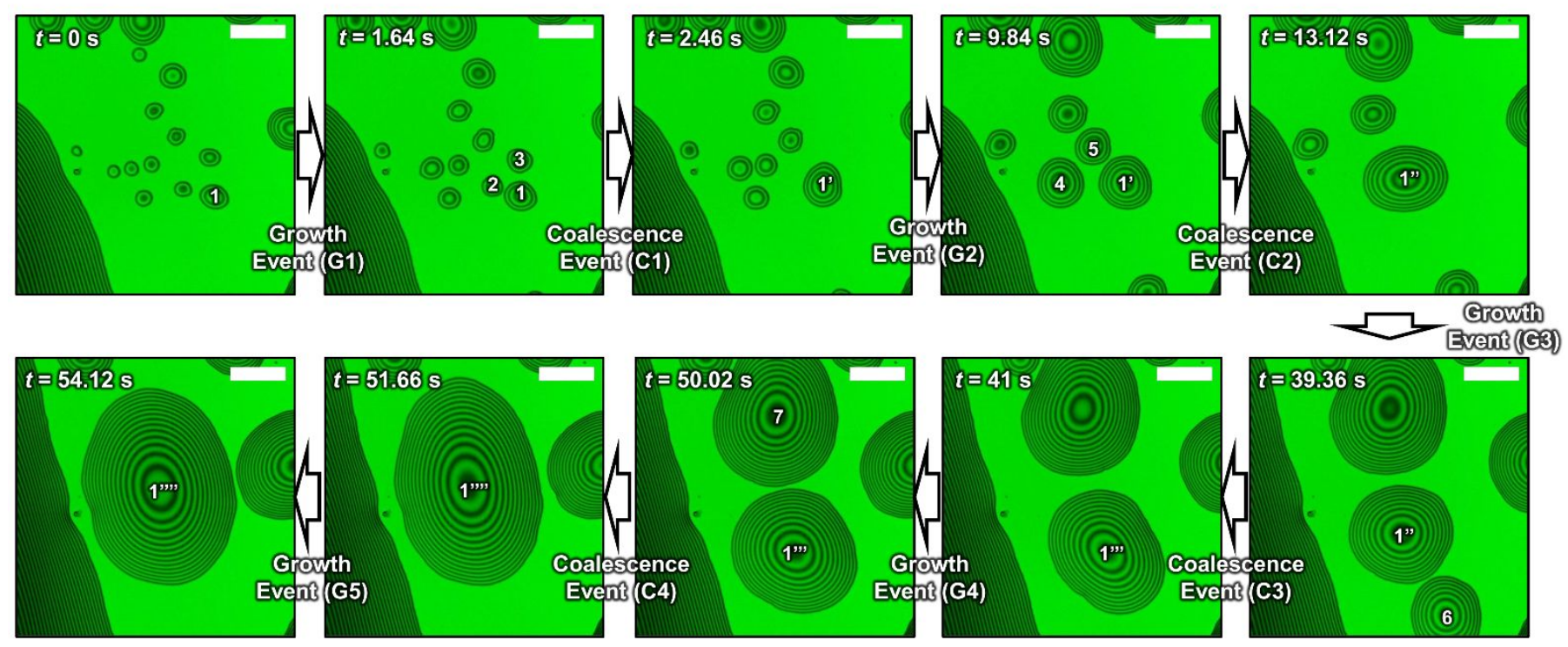

Figure S1. Time evolution of a typical acetone microdroplet condensation event (observation set \#2).

During the observation period of $54.12 \mathrm{~s}$, the droplet of interest (denoted by 1 ) underwent five such growth events (namely, G1, G2, G3, G4 and G5) and four coalescence events (namely, C1, C2, C3 and C4). The droplet number is kept the same during the pure growth phases while it has been updated after each coalescence. During C1, droplet numbered 1, merges with droplets numbered 2 and 3 to form droplet numbered 1'. Similarly, droplet 1' merges with droplets 4 and 5 to from droplet 1', during C2. Droplets numbered 1", and 6 undergo coalescence thereafter to form droplet 1"', during C3. Following this, droplets 1 ", and 7 merge to form droplet 1",' during C4. The scale bar is $50 \mu \mathrm{m}$. The corresponding experimental dynamics is presented in the form of a time-series of simultaneously captured dual-wavelength interferograms in supporting video $\mathrm{S} 2$, with identically marked events. 


\section{Supplementary note 2}

\section{Quantitative analysis of growth and coalescence of a condensed acetone microdroplet -}

\section{observation set \#2}

To assess the consistency of our observed quantitative trend for observation set \#1 (Figure 4), we carry out similar quantitative analysis of the experimental data for observation set \#2 as well. The results are illustrated in Figure S2. As can be seen in Figure S2A, volume, $V$ and liquid-vapor surface area, $S_{l v}$ follow similar scaling relationship with mean base radius, $r_{\text {mean }}$ as the ones reported in Figure 4A. For observation set \#2, we obtain $S_{l v} \sim r_{\text {mean }}{ }^{1.98}$ and $V \sim r_{\text {mean }}{ }^{2.98}$ by carrying out a power-law fitting to the experimental data, which stand in excellent agreement with the analytical formulas ${ }^{17}$ for an ideal spherical cap where $S_{l v} \sim r_{\text {mean }}{ }^{2}$ and $V \sim r_{\text {mean }}{ }^{3}$. It implies that the spherical cap approximation is consistent and can be applied for observation set \#2 as well.

Further, the values of mean contact angle, $\theta$, have also been plotted against $r_{\text {mean }}$ in Figure S2B. In observation set $\# 2$, the contact angle values remained almost constant (within $3.25^{\circ}-4.75^{\circ}$ ) and the values were effectively invariant with respect to changes of the base radii, similar to the reported trend for observation set \#1 (Figure 4B).

Additionally, we have also captured the experimentally observed trend of growth of mean base radius $r_{\text {mean }}$ with time in Figure S2C and compared it to the theoretical prediction of Rykaczewski ${ }^{4}$ for droplet growth in constant contact angle mode, given by Eq. (6) of the main manuscript. For observation set $\# 2, \phi$ is assumed to be $3.96^{\circ}$ as the time averaged value of mean contact angle over the entire observation window of $54.12 \mathrm{~s}$ was $3.96^{\circ}$. As shown in Figure S2C, we have obtained fitted values of $\Delta T$ in the range of $(9.5-14 \mathrm{mK})$ for the observation set $\# 2$ which is comparable with that of the observation set \#1 (7.5-25 mK). 

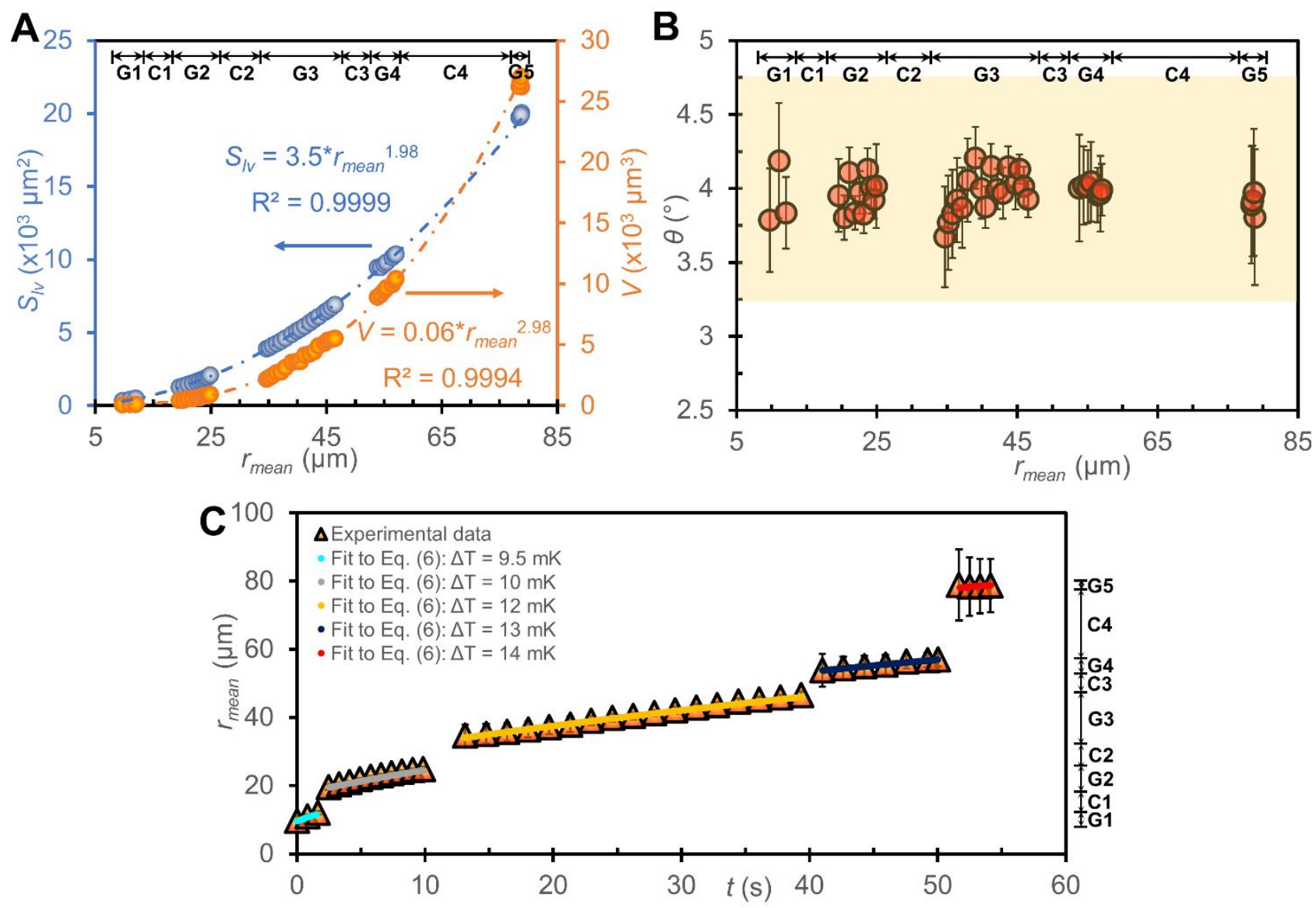

Figure S2. Quantitative analysis of the dynamics of growth of a condensing acetone microdroplet

corresponding to observation set \#2. (A) Variation of droplet volume $V$ and liquid-vapor surface area

$S_{I V}$ with mean base radius. (B) Dependence of the mean contact angle value on mean base radius. A

total of 360 local contact angles were averaged across the contact line of the droplet at each instance.

The error bars represent the standard deviation of these 360 local values. (C) The evolution of mean

base radius $r_{\text {mean }}$ with time during condensation. Similar to Figure S2B, a total of 360 local values of base radii were considered along the 180 different slicing direction with $1^{\circ}$ angular interval to evaluate the mean base radius. The error bars represent the standard deviation of these 360 local base radius 
values. The presented plots characterize the evolution of the droplet denoted by 1 in Figure S1 and supporting video S2, with G1, G2, G3, G4 and G5 indicating the individual growth events and C1, $\mathrm{C} 2, \mathrm{C} 3$ and $\mathrm{C} 4$ denoting the coalescence events. 